\title{
Targets, targeted targets and molecular imaging: new potential treatments, new treatments and new ways of assessing treatments
}

\author{
Peter Harper
}

Received: 10 September 2009 / Accepted: 11 September 2009/Published online: 30 October 2009

(C) Springer-Verlag 2009

Over decades as a medical oncologist, dramatic changes have occurred in what we can achieve. This has most recently come from an understanding of targets and in this issue we are looking at how to image those changes.

In some diseases the discovery of the target predated by several decades the discovery of the targeted treatment. In others targeted treatments were developed and only latterly have we understood which population is likely to be targeted.

An example of the former is the discovery of the Philadelphia Chromosome, a fusion of two gene products leading to the BCR-ABL gene. The BCR-ABL gene leads to the production of the BCL-ABL tyrosine kinase and growth stimulation, hence chronic myologenous leukaemia. The targeting of this pathway by inhibitors of tyrosine kinase had led to long-term control of the disease.

In lung cancer the targeted drug (EGFR receptor blocker) was discovered before we found which group of patients would respond better and why they would respond better. Essentially, in multiple phase II studies and phase III randomized trials, a series of clinical parameters were associated with clinical responsiveness to the EGFR blockers. These included adenocarcinoma (particularly bronchioalveolar carcinoma) female, non-smokers and Asian origin. Many of these clinical parameters proved to be mediated through differences in the frequency of the EGFR and KRAS mutations. The discovery of these mutations in the tyrosine kinase domain of the EGFR (exon 19 deletion,

\section{P. Harper $(\bowtie)$}

Medical Oncology, Guy's Hospital,

3rd Floor, Thomas Guy House, St. Thomas Street,

London SE1 9RT, UK

e-mail: peter.harper@kcl.ac.uk
L858R point mutation in exon 21) has subsequently been found to delineate a subset of patients who are much more likely to respond to treatment and a population who are most unlikely to respond to such EGFR blockers.

In this issue my co-editor, Professor Tony $\mathrm{Ng}$, and his co-authors have set out the newer imaging techniques, with an emphasis on response to targeted treatments.

Josephs, Spicer and O'Doherty discuss molecular imaging in clinical trials. The exponential rise in the cost of clinical trials they say mandates that to continue to develop anticancer treatment, we need to gain early signals of their efficacy. Such early signals may encourage the developers ("big pharma" or "biotech") to continue along their current pathway, change that pathway and even abandon the approach. We do, therefore, need to understand these signals and "get them right". The molecular imaging used to explore many of the hallmarks of cancer biology, including angiogenesis, proliferation, tissue invasion, evasion of apoptosis and self-sufficiency in growth signal can all be imaged using a combination of single photon emission computed tomography (SPECT), positron emission tomography (PET), magnetic resonance imaging (MRI), and optical techniques. The availability of various isotopes with varying physical properties particularly suits PET imaging as a functional modality for application in clinical trials. Priorities for the development of imaging are also discussed.

Gunasekera, Pankhurst and Douek discuss imaging applications of nanotechnology. They speculate and preview the concept of a single agent capable of diagnosing cancer, treating cancer and monitoring the response to that treatment. They review the advances in nanotechnology, bringing the clinician closer to the development of dual and multi-functional nanoparticles, challenging the individual distinction between diagnostic agents and treatment agents. 
They review and ask us to question whether a fresh look should be taken at the clinical challenges and the way nanotechnology can address them.

Torres Martin de Rosales, Blower and Årstad review nuclear imaging of molecular processes. By the use of radio tracers (isotopes) at very low concentration (the concentrations are low enough not to perturbate the actual processes), to explore peptide receptor expression, angiogenesis, multi-drug resistance, hypoxia, and glucose metabolism. They review the imaging techniques PET and single photon radionuclide imaging with molecules labeled with gamma and positron imaging radioisotopes.

Dancey, Begent and Meyer review imaging for targeted therapeutics. Antibodies have high tumor specificity and can be engineered to optimize delivery to, and retention within, the tumor. Whole antibodies can activate natural immune effector mechanisms and can be conjugated to beta and alphaemitting radionuclides, toxins, enzymes and nano-particles for an enhanced therapeutic effect. Imaging, they report, is central to the development of these agents and is used for patient selection, for performing dosimetry and for assessment of response. Iodine-131 perhaps all clinicians have experience with, but the newer indium-111 octreotide images and yttrium-90 octreotate images are becoming more known to us from the treatment of neuroendocrine tumors. The authors review the diverse data that has been acquired.

Enfield, Gibson, Hebden and Douek review optical tomography of breast cancer. Optical imaging and spectroscopy use near-infrared light to derive physiological parameters such as total hemoglobin concentration and tissue oxygen saturation. Optical imaging can distinguish between benign and malignant tissue and can be used for monitoring treatment response, providing functional and dynamic imaging to reflect the changes in tumor angiogenesis and hypoxia. Using neo-adjuvant chemotherapy as an example in breast cancer, they described their work. It appears possible to predict a response to treatment within
4 days of initiation of that treatment. Combining optimal measurements with PET, standard radiology, MRI and ultrasound, are all methods we are used to. Optical tomography may well be an addition to this.

Finally in the section Perspective, Kelleher et al review the potential of optical proteomic technologies to individualize prognosis and to guide rational treatment for cancer patients. Translational genomic and proteomic research is used to discriminate between patient outcomes, develop rational treatments, and target specific molecular pathways of the individual tumor. Tailoring that treatment to that patient's risk is the next step. It has been an oncologists dream for decades to target the right drug to the right tumor. Optical proteomics are new to most clinicians. FRET, FLIM and tissue microarrays may well become part of our everyday language. An understanding of translational modifications, such as phosphorylation and ubiquitination can be performed in both cell lines and with great efficiency in cancer tissue samples using tissue microarrays. The concept of response to the treatment at a molecular level can be monitored during treatments. Re-biopsy may become part of normal therapeutic assessment. Biopsies may prove to be both "prognostic" of individual risk and "predictive" of response to "selected" targeted anticancer treatments.

We have come a long way in targeted treatments. As mentioned in the introduction, from Philadelphia Chromosome now having proved to be a target for specific tyrosine kinase inhibitors to a selection of patients with adenocarcinoma of the lung for EGFR inhibitors. Along the way we also had targeted treatment for gastrointestinal stromal tumors and of course targeted treatments of a wider usefulness including bevacizumab.

In this issue of Targeted Oncology, perhaps we will, through better imaging and understanding of imaging, see where we are going in developing further targets and targeted treatment. 